The effect of recent reminder setting on subsequent strategy and performance in a prospective memory task

Chiara Scarampi* and Sam J. Gilbert ${ }^{\mathrm{a}}$

${ }^{a}$ Institute of Cognitive Neuroscience, University College London, United Kingdom

Address correspondence to:

Chiara Scarampi

Institute of Cognitive Neuroscience

17 Queen Square

London WC1N 3AR

UK

Email: c.scarampi@ucl.ac.uk

Tel: +44 (0)20 76791199 


\section{The effect of recent reminder setting on subsequent strategy and performance in a prospective memory task}

The technological advancement that is rapidly taking place in today's society allows increased opportunity for "cognitive offloading" by storing information in external devices rather than relying on internal memory. This opens the way to fundamental questions regarding the interplay between internal and external memory and the potential benefits and costs of placing information in the external environment. This article reports the results of three pre-registered online experiments investigating the consequences of prior cognitive offloading on A) subsequent unaided ability, and B) strategic decisions whether to engage in future cognitive offloading. We administered a web-based task requiring participants to remember delayed intentions for a brief period and manipulated the possibility of setting reminders to create an external cue. Earlier cognitive offloading had little effect upon individuals' subsequent unaided ability, leading to a small and nonsignificant drop in subsequent performance. However, there was a strong effect on participants' subsequent likelihood of setting reminders. These findings suggest that the short-term impact of cognitive offloading is more likely to be seen on individuals' strategy choices rather than basic memory processes.

Keywords: Prospective Memory; Cognitive Offloading; Technology; Reminders; Delayed Intentions; Strategy Use

\section{Introduction}

In everyday life, we often form intentions for future actions which can only be executed after a delay. However, our mental abilities have acknowledged limits (e.g., we can encode and store in memory a limited amount of information; Cowan, 2010), so that we sometimes forget to execute an intended behaviour. When establishing an intention to act later, we can alternatively choose to use physical actions (e.g., setting a reminder such as an alarm or calendar alert) as a means of "cognitive offloading" (Risko \& Gilbert, 2016) so that the information processing requirements of the task are altered. This in turns reduces cognitive demand and relieves the burden of intention 
maintenance in internal memory (Herrmann, Brubaker, Yoder, Sheets, \& Tio, 1999).

More precisely, the use of external resources allows us to reserve the limited cognitive resources for the information that cannot be accessed through external means (Macias, Yung, Hemmer, \& Kidd, 2015).

Although the phenomenon is ancient, researchers have only recently started conducting systematic investigations on the topic. This has been fuelled by rapid technological advancement, which allows us to use the environment (i.e., external memory) to record information rather than storing it in our brain (i.e., internal memory; Finley, Naaz, \& Goh, 2018). Researchers have started questioning what the effects of cognitive offloading are on our future thinking and behaviour. In the current study, we explore the effects of recent use of reminders on subsequent performance and strategy in a task requiring memory for delayed intentions ${ }^{1}$. More precisely, we study the effect that reminder setting (also known as "intention offloading”; Gilbert, 2015a) has on unaided memory ability and whether it influences the propensity to use the same strategy for future performance.

\section{The relationship between intention offloading and unaided memory}

While some experiments have shown that offloading information can improve performance when the external store is available (see Risko \& Gilbert, 2016), other research has documented other potential effects of relying on external devices on cognition. Recent studies have shown that using the Internet to search for information can lead to blurred boundaries between individuals' own minds and the mind of the

\footnotetext{
${ }^{1}$ Our paradigm requires "prospective memory" (Einstein \& McDaniel, 1990; Smith, 2003) in the broad sense that participants need to remember delayed intentions, albeit over a very short delay. However, some authors use the term more narrowly to refer to situations where intentions are maintained over longer periods and must be brought back to mind at the appropriate moment (Graf \& Uttl, 2001). Our paradigm would correspond to the former sense but not the latter.
} 
internet (Fisher, Goddu, \& Keil, 2015; Ward, 2013). Fisher and colleagues (2015) documented an illusion of knowledge by which individuals using the Internet to search for information tend to conflate information available online with information stored in their own memory. In a similar vein, Ward (2013) found that using the internet causes people to assimilate its attributes into the self, overlooking the effect of the internet on performance and inflating judgements about the ability to perform well in the future.

Other research has documented instances where relying on external memory may prevent individuals from maintaining an internal representation of the information they are attempting to preserve (Henkel, 2014; Soares \& Storm, 2018; Tamir, Templeton, Ward, \& Zaki, 2018). Henkel (2014) documented a photo-takingimpairment effect by which participants may be less likely to remember objects they photograph rather than objects they simply observe. Photography can be interpreted as a form of cognitive offloading, which allows individuals to rely on the external device to record information about the object, rather than storing it in the internal memory. This result has been recently replicated by Soares and Storm (2018), who found that participants exhibit a photo-taking-impairment effect even when they do not expect to have subsequently access to the photos. Converging support has been provided by a further study showing that media usage can impair memory (Tamir et al., 2018). Altogether, these findings suggest that the act of cognitive offloading may have a detrimental effect on internal memory for the offloaded information. However, these photo and media impairment effects refer to the impact of offloading on unaided memory for the specific memories that were offloaded. Equally interesting is the investigation of the effect of earlier offloading of some information on subsequent unaided memory for different information. 
A common concern is that relying on cognitive aids can hinder unaided ability (Baldwin, Powell, \& Lorenc, 2011) by reducing opportunities for individuals to develop and maintain the skills required to perform tasks in an unaided manner (i.e., 'use it or lose it'). According to this view, simplifying a task by adopting a cognitive offloading strategy would lead to impaired subsequent performance on a more demanding task (i.e. one which does not afford offloading).

An alternative view is offered by the literature on ego depletion (see Friese, Loschelder, Gieseler, Frankenbach, \& Inzlicht, 2018 for a review). Although the literature in the field is controversial, previous research has suggested that initially performing a demanding self-control task can lead to impaired subsequent cognitive performance, compared with initial performance of an easier task. While some authors propose that this is because self-control is a depletable resource (Baumeister, Bratslavsky, Muraven, \& Tice, 1998), others have challenged this view both conceptually (Lurquin \& Miyake, 2017) and empirically (Hagger et al., 2016). For the present purposes, we simply note that the ego depletion literature makes an opposite prediction to the "use it or lose it" account, namely that performing a simplified task by adopting a cognitive offloading strategy might improve subsequent cognitive performance in a demanding task rather than impairing it. This prediction does not imply acceptance of the concept of resource depletion and would be equally compatible with other models of ego depletion (e.g. the idea that these effects reflect participants' beliefs and motivational states, rather than self-control being a limited resource).

Previous work has investigated the effect that saving a file on a computer has on the remembering of new information (Storm \& Stone, 2015). Not only is the proportion of information recalled from the saved file higher than the information recalled when the file is not saved, but also, saving a file before studying a new file improves recall of 
the contents of the new file. That is, saving files represents a means to offload to-be remembered information onto outside sources, which facilitates the later encoding and remembering of new information. This effect suggests that offloading to-beremembered information can improve learning of subsequent information. This finding was replicated in a recent study by Runge Frings, and Tempel (2019) who also investigated whether memory offloading can be beneficial for subsequent cognitive demanding tasks in general by examining performance in unrelated arithmetic tasks as a function of the possibility to save (i.e., offload) an earlier learned word list. Not only did participants who saved the word list have a better recall of a new word list, but they also performed better in an unrelated arithmetic task. These results suggest that the benefits of memory offloading are not limited to memory performance but can free cognitive resources that can be used for subsequent tasks. Of the two accounts described above, this would be more compatible with the ego depletion model. However, in the paradigm used by Storm and Stone and Runge and colleagues, participants must learn the second file whilst still needing to remember the first one, potentially leading to interference and competition between the two files when the first one is not offloaded.

By contrast, in this study we were interested in investigating whether initially adopting an offloading or a non-offloading strategy affects subsequent memory performance, even when the original material has already been tested and is no longer relevant. In other words, we tested whether cognitive offloading at time A affects cognitive performance at time $\mathrm{B}$, even when the material learned at time $\mathrm{A}$ is no longer relevant in the subsequent phase of the task. We did this in Experiment 1 by adapting the intention offloading paradigm used by Gilbert (2015a). Participants performed a memory test in two phases. In the first phase participants were randomised into two groups performing the task either with external reminders or unaided memory. In the 
second phase, all participants performed using unaided memory. This allowed us to test the impact (if any) of using external reminders on subsequent unaided memory performance.

\section{The relationship between previous reminder setting and subsequent strategy choice}

Beside understanding of the consequences of cognitive offloading on our unaided memory ability, another fundamental issue is the investigation of the factors that affect the likelihood of choosing to offload cognition rather than rely on the internal memory. In recent years, researchers have focused on different factors that affect cognitive offloading, such as task difficulty (Gilbert, 2015a), metacognitive beliefs (Gilbert, 2015b), age (Gilbert, 2015a), task instructions (Boldt \& Gilbert, 2019), feedback valence and practice-trial difficulty (Gilbert et al., 2019). A further potential factor that has not been studied until now is participants' past history and previous experience with the act of cognitive offloading.

A growing body of research has shown that when solving a problem, individuals are inclined to reuse the strategy they used before (Luchins, 1942; Schillemans, Luwel, Bulté, Onghena, \& Verschaffel, 2010). This effect has been demonstrated in several domains ranging from anagrams (Ellis \& Reingold, 2014) to perceptual decisionmaking (Schillemans et al., 2010) and chess (Bilalić, McLeod, \& Gobet, 2008). A similar perseveration effect has been found when individuals use the Internet as a form of cognitive offloading (Ferguson, McLean, \& Risko, 2015; Storm, Stone, \& Benjamin, 2016). Ferguson and colleagues (2015) found that access to the Internet influences individuals' willingness to volunteer answers, leading to fewer correct answers overall but greater accuracy when an answer was offered. In a similar vein, Storm and colleagues (2016) found that using Google to answer an initial set of trivia questions 
make individuals more likely to rely on the Internet to answer a new, relatively easier, set of questions. This suggests that once individuals are in the habit of using the Internet, they keep using it, even when it is not needed.

Starting from these observations that behaviour is influenced by the strategies previously used, another aim of this study was to test the hypothesis that the use of reminders in an offloading task increases the likelihood of relying on this strategy in the future. We did this in Experiments 2 and 3 by adapting the intention offloading paradigm used by Gilbert (2015a). Participants performed a memory test in two phases. In the first phase participants were randomised into two groups performing the task either with external reminders or unaided memory. In the second phase, all participants were given the opportunity to choose whether to use reminders or rely on their own memory. This allowed us to test whether once people are in the habit of using reminders, they keep using them.

\section{Experiment 1}

This experiment aimed to investigate whether initially adopting an offloading or a nonoffloading strategy affects subsequent unaided memory performance, even when the original material has already been tested and is no-longer-relevant.

We evaluated the evidence for the following three alternative hypotheses:

- The use of external reminders impairs subsequent unaided memory performance, as predicted by the 'use it or lose it' account.

- The use of reminders improves subsequent unaided ability to execute a future intention, as predicted by the 'ego depletion' account.

- The use of external reminders has no impact on subsequent unaided memory performance. 
Before commencing data collection, we preregistered our hypotheses, sample size, experimental procedure, participant exclusion criteria, and analysis plans (https://osf.io/724x5/).

\section{Method}

\section{Participants}

A total of 220 participants were recruited from the Amazon Mechanical Turk website (http://www.mturk.com), an online marketplace in which participants receive payment for completion of web-based tasks (Crump, McDonnell, \& Gureckis, 2013).

A statistical power analysis was performed with $\mathrm{G}^{*}$ Power 3.1 for sample size estimation. To the best of our knowledge, no previous studies have investigated the effect of reminder use on subsequent unaided memory. As a consequence, for the purpose of sample size estimation, we referred to the literature on ego depletion, as similarly to our experiment, the typical paradigm used to test ego depletion consists of two conditions, both requiring participants to complete two consecutive tasks. We based the computations on a recent meta-analysis of the ego depletion effect conducted by Dang (2018). Since none of the tasks used in the ego depletion literature is comparable to that used in our experiment (i.e., a delayed intention task coupled with an arithmetic interruption question), we referred to the overall effect found in the meta-analysis: $g=$ 0.38. With an alpha $=.05$ and power $=0.80$, the projected sample size needed with this effect size was approximately $N=220$ for the simplest between group comparison (110 participants in each group).

As in earlier studies by Gilbert (2015a, 2015b), participation was restricted to volunteers aged at least 18 years and living in the USA, to reduce heterogeneity. Furthermore, participants not achieving a criterion of at least $80 \%$ accuracy in the 
arithmetic-verification test or $50 \%$ accuracy in the intention-offloading task were excluded and replaced.

Of the resulting sample, 129 were male and 91 female, with a mean age of 36 years (range 19-70). Participation took on average 25 minutes (minimum $=8$ minutes, maximum $=3$ hours 12 minutes), for which participants were paid $\$ 2.50$. Ethical approval was received from the local ethics committee and participants provided informed consent before participating in the study.

\section{Task and Procedure}

The task was programmed in Java using Google Web Toolkit version 2.8 (http://www.gwtproject.org) and Lienzo graphics toolbox version 2.0 (http://emitrom.com/lienzo), implemented in the Eclipse development environment (https://www.eclipse.org). The experiment code needed to replicate the current study can be found by visiting the first author's account on Open Science Framework (https://osf.io/3f52c/).

Intention-offloading task. In each trial, ten yellow circles numbered 1 to 10 were randomly positioned within a square. Participants were instructed to use the mouse to drag the circles in ascending order $(1,2,3$, etc. $)$ to the bottom of the square. Each time a square was dragged to the bottom, it disappeared, leaving the other circles on the screen. After the 10th circle disappeared, the screen was cleared and the next trial began (see Figure 1 for a schematic illustration of the task and visit http://www.ucl.ac.uk/samgilbert/demos/circleDemo.html for a demonstration).

Alongside this ongoing task, on each trial participants were provided with delayed intentions. That is, they were instructed to drag three circles to a specific alternative location (i.e. left, right, or top of the square) when the numbers were reached 
in the sequence. This led to the formation of delayed intentions to perform particular actions when they encountered prespecified cues, although participants could produce a standard ongoing response (i.e., dragging the circle to the bottom of the box) if they forgot.

The task also permits intention offloading in a simple manner: at the beginning of each trial, participants can drag the target circles towards their intended location. From this point on, there is no need to mentally rehearse the delayed intention(s). Instead, the locations of the target circles themselves represent the intention, providing a perceptual trigger when they are reached in the sequence. An everyday analogue might be leaving an object by the front door, so that we remember to take it with us when leaving the house. [Figure 1 approximately here]

There are three potential intention offloading conditions in this paradigm. In the No Reminders condition, participants rely on internal memory only. This is enforced by fixing the positions of all circles on screen apart from the next in the sequence, so that it is not possible to adjust the position of forthcoming targets when they first appear on screen. In the Forced Reminders condition, participants are required to set external reminders. This is enforced by preventing participants from continuing the ongoing task (removing circles from the bottom of the box) until they have adjusted the position of any new target circles that appear. In the Optional Reminders condition (used in Experiments 2 and 3), participants have a free choice whether or not to set reminders.

Arithmetic-verification test. Participants in both conditions additionally received a distracting arithmetic question during each trial, via a pop-up box (using the same procedure as Gilbert, 2015a). This occurred immediately after dragging one of the nontarget circles to the bottom of the box, at a position in the sequence randomly selected between the first circle and the circle immediately before the first target. The 
purpose of this was to increase the difficulty of the task, helping to reduce the possibility of ceiling effects in the measure of intention fulfilment.

Manipulation. The experiment consisted of 20 experimental trials, divided in two phases and the key element of the study was the manipulation of intention offloading in the first phase (See Figure 2 for a schematic representation of the experimental design). [Figure 2 approximately here] More precisely, at the beginning of the experiment, participants were randomly assigned to one of two conditions: No Reminders or Forced Reminders. Participants in the No Reminders condition were asked to complete the intention offloading task relying on their internal memory only, whereas participants in the Forced Reminders condition were asked to complete the task making use of reminders.

A second phase of the experiment followed immediately after the first and was identical for participants in both conditions. That is, they were given 10 trials of the same intention-offloading task, but this time none of them was allowed to set reminders.

\section{Apparatus}

Participants completed the task via their computer's web browser. As in Gilbert (2015a), participation was only permitted if the browser window had dimensions of at least $500 \times 500$ pixels. The square box containing the circles was sized at $80 \%$ of the horizontal or vertical extent of the browser window, whichever was smaller. Each circle had a radius of $5.5 \%$ of the width/height of the box, and all circles were initially placed so that they fell within a central portion of the box with dimensions sized at $56 \%$ of the total width/height, so that no circles were adjacent to any of the edges of the box at the beginning of the trial. 


\section{Data Analysis}

All data analyses were conducted in $\mathrm{R}$ version 3.5.2. Bayesian independent samples ttests were conducted in JASP version 0.9.2. The key dependent variable was target accuracy - i.e., the proportion of targets that are correctly dragged to the instructed location rather than to the bottom of the square.

\section{Results}

Mean arithmetic-verification accuracy was 99\%. We first compared accuracy between the two groups in phase 1, using a Welsh two-sample t-test. Accuracy was significantly higher in the Forced Reminders group than the No Reminders group, $t(210.07)=3.69, p$ $<.001, d=0.50$ showing that the use of external reminders improves task performance compared to the use of internal memory only (see Figure 3). [Figure 3 approximately here]

Next, we investigated the effect of using reminders in the first phase on unaided memory ability in the second phase. This was our main hypothesis-testing analysis. An independent sample t-test revealed no reliable difference between the two groups, $t(218)$ $=1.03, p=.30, d=0.14$. We also performed some Bayesian hypothesis testing to further quantify the support in favour of the hypotheses again each other. We conducted three separate Bayesian t-test tests, corresponding to each of the three hypotheses above. A first test evaluating the evidence in favour of the hypothesis that the use of external reminders impairs subsequent unaided prospective memory performance ('use it or lose it' account) yielded a BF of 2.47 in favour of the null hypothesis that there is no effect of cognitive offloading on subsequent unaided memory ability. According to Jeffreys's (1961) classification scheme, this result can be interpreted as anecdotal evidence for the null hypothesis. A second test evaluating the evidence in favour of the 
hypothesis that the use of reminders improves subsequent unaided ability to execute a future intention ('ego depletion' account) yielded a BF of 12.92 in favour of the null hypothesis of no effect of reminder setting on unaided ability. This result can be interpreted as strong evidence in favour of the null hypothesis. Finally, a third test evaluating the evidence in favour of the hypothesis that use of external reminders has no impact on subsequent unaided memory performance yielded a BF of 4.14 in favour of the hypothesis that the No Reminders group and the Forced Reminders group can be best described by the same distribution.

\section{Discussion}

The results replicated the finding that individuals who set reminders fulfil their delayed intentions more often (Gilbert, 2015a). Furthermore, unaided memory ability in phase 2 did not significantly differ in the No Reminder and Forced Reminder group. This suggests that prior use of a cognitive offloading strategy does not have a strong impact on subsequent unaided memory ability, at least over the short time-scale investigated in this experiment. More precisely, the data provides strong evidence against the hypothesis that the use of reminders improves subsequent unaided ability to execute a future intention ('ego depletion' account). Conversely, despite the lack of significant differences between the two groups, the evidence only provides anecdotal evidence in favour of the null hypothesis. Therefore, our results seem compatible with the idea that prior reminder setting has no impact on subsequent unaided performance, or a small detrimental effect (e.g., because it reduced practice opportunities; cf Baldwin et al., 2011). However, the possibility of a detrimental effect rests on a nonsignificant finding, so it is not strongly supported. Of course, our results can only speak to the impact of offloading on a short timescale, seeing as the total experiment duration was only about 25 minutes. It is quite possible that impact over a longer timescale could be different. 
Our results contrast with previous evidence showing that prior offloading can improve subsequent unaided memory (e.g., Storm \& Stone, 2015). We suggest that the key difference is that in Storm and Stone's study, participants needed to maintain the original information (either in internal memory or an external store) while the subsequent memory test took place. Thus, participants had to learn new information whilst simultaneously maintaining earlier information either in internal memory or an external store. By contrast, in our study the earlier memoranda were no longer relevant at the time of the subsequent memory test. Therefore, regardless of whether the original information was stored internally or externally, it could be dismissed from memory by the time the subsequent memory test took place.

In summary, our results suggest that short-term use of a cognitive offloading strategy has little effect on memory ability in circumstances where the previously memorised information is no longer required and participants are forced to use unaided memory. We now turn to the question of whether prior cognitive offloading influences subsequent behaviour when participants are given a choice whether or not to set reminders.

\section{Experiment 2}

In Experiment 1, we investigated whether initially adopting an offloading versus a nonoffloading strategy affects subsequent memory performance when participants are prevented from setting reminders. However, in most everyday life situations, individuals are free to choose whether or not to use a cognitive offloading strategy to reduce the cognitive demands of a task (Risko \& Gilbert, 2016). This experiment aimed at understanding whether the use of a cognitive offloading strategy becomes habitual. We did this by adapting the intention offloading paradigm used in the previous experiment. Again, participants performed a memory test in two phases. In the first 
phase they were randomised into groups performing the task either with external reminders or unaided memory. In the second phase, all participants were given a free choice whether to use reminders or rely on their own memory.

We predicted that participants who were instructed to set reminders in the first phase of the experiment would rely more on reminders in the second phase than would participants who were instructed to solve the task using their own memory in phase 1. The use of reminders may affect not only the likelihood of using them again in the future, but also performance accuracy. We expected to replicate the finding that the use of reminders increases performance accuracy. Accordingly, we predicted that in phase 1 participants who were initially instructed to set reminders would be more accurate than participants who were initially asked to solve the task using their own memory.

Additionally, we hypothesised that a sufficiently-powered study would show higher accuracy in phase 2 for participants who initially used reminders compared with those who initially used their own memory, due to increased use of reminders in the former group. However, our study was not well powered for this particular test (see below), so we hypothesised that in phase-2 accuracy would either be significantly higher in the reminder group, or not significantly different between the two groups.

Before commencing data collection, we preregistered our hypotheses, sample size, experimental procedure, participant exclusion criteria and analysis plans (https://osf.io/9wtd8/).

\section{Method}

\section{Participants}

A total of 192 participants took part in the experiment (mean age: 35 years, range: 19$73 ; 70$ female, 122 male). This was based on a power calculation aiming for $80 \%$ power 
to detect a between-group difference with effect size $d=.41$, based on unpublished pilot data (see https://osf.io/9wtd8/ for further information). This was our predicted effect size for the difference in reminder setting between groups who had previously performed the Forced Reminders versus the No Reminders versions of the task.

We also performed a power analysis to identify the sample size that would be required to detect a putative difference in phase- 2 accuracy between the groups, seeing as a group that set more reminders would also be predicted to achieve higher accuracy. The computations were based on the results of Experiment 1, which revealed an effect size of $d=0.498$ when comparing accuracy in a group of participants who always used reminders with those who never did. However, we expect that the effect of our group manipulation on phase- 2 reminder use will be much smaller in magnitude when participants have a free choice whether or not to use reminders. For example, suppose that the difference between the two groups was $20 \%$ (e.g., $60 \%$ reminder use in one group, $40 \%$ in the other). This might predict an effect one-fifth of the size of the earlier study, which would require a sample size of $N=3164$ (1583 participants in each group). Seeing as this is much larger than our proposed sample size, we do not believe that our study would be well-powered for this comparison (as stated in the original preregistration), and an alternative task where there is a stronger relationship between reminder-use and accuracy might be more appropriate to investigate this question. Participants were randomly allocated to two groups: Forced Reminders (96 participants) and No Reminders (96 participants). The same exclusion criteria as in experiment 1 were used. Participation took on average 22 minutes (minimum $=8$ minutes, maximum $=2$ hours 35 minutes), for which participants were paid $\$ 2.50$. All participants provided informed consent before participating and the research was approved by the UCL Research Ethics Committee. 


\section{Data Analysis}

The analyses focused on two key dependent measures: target accuracy - i.e., the proportion of targets that are correctly dragged to the instructed location rather than to the bottom of the square - and externalising proportion - i.e., the proportion of target circles for which participants set up an external reminder, by moving it to a different location before reaching its position in the ongoing task. For each trial, a record was made of any time that a participant clicked on a circle when it was not next in sequence (e.g. clicking on circle number 5 , when circle number 1 is still on the screen). The proportion of targets clicked before their turn in the sequence was then calculated, by dividing the number of target circles that were clicked when it was not their turn in the sequence by the total number of targets (i.e., three). An analogous procedure was used to calculate the proportion of nontarget circles that were clicked out of sequence. This was subtracted from the target-circle proportion, to control for any out-of-sequence clicks that might have occurred simply due to accidentally clicking on the wrong circle, regardless of its status as target or nontarget. This yielded the externalizing proportion, calculated so that 0 would indicate that participants did not click on target circles out of sequence any more often than nontarget circles, and where 1 would indicate that participants clicked every target circle before reaching its position in the sequence, but never clicked on nontarget circles in this way.

\section{Results}

Mean arithmetic-verification accuracy was $99 \%$.

\section{Objective Accuracy}

We first compared accuracy between the two groups in phase 1, using a Welsh twosample t-test. Accuracy was significantly higher in the Forced Reminders group than the 
No Reminders group, $t(180.39)=4.39, p<.001, d=0.63$ showing, as in the previous experiment, that the use of external reminders improves task performance compared with the use of internal memory only (see Figure 3).

Next, we conducted an independent samples t-test comparing accuracy in phase 2 between the two groups. We did not find any significant difference between the two groups, $t(190)=0.06, p=.95, d=0.01$. Finally, we compared accuracy in each group between phase 1 and phase 2, using a paired t-test. Results showed a marginally significant decrease from phase 1 to phase 2 in the Forced Reminders group $(t(95)=1.95, p=.053, d=0.20)$ and highly significant increase in the No Reminders group $(t(95)=3.34, p=.001, d=0.34)$.

\section{Reminder Setting}

As our main hypothesis-testing analysis, we investigated the effect of using reminders in the first phase on the likelihood of using reminders in phase 2. A Welsh two-sample t-test revealed a significantly higher externalisation proportion in the Forced Reminders group, $t(183.29)=4.48, p<.001, d=0.65$ (see Figure 4). [Figure 4 approximately here]

A Bayesian independent samples t-test was also conducted to investigate the strength of the evidence in favour of our hypothesis against the null hypothesis. An estimated Bayes factor (null/alternative) suggested that the data were 1315 times more likely to occur under a model including an effect for experimental condition, rather than the model without it.

\section{Relationship between Accuracy and Reminder Setting}

Next, we ran a correlation between accuracy in phase 1 and reminder choice in phase 2 to investigate whether there is a relationship between performance in phase 1 and the choice to set reminders in phase 2 . We found a significant positive association between 
the two variables for the Forced Reminder group, $r_{\mathrm{s}}(94)=.28, p=.006$, and a significant negative correlation for the No Reminders group, $r_{s}(94)=-.25, p=.016$.

We also combined over the two groups and ran a multiple regression where group was a factor. The results of the regression indicated the two predictors explained $10 \%$ of the variance $\left(R^{2}=.10, F(2,189)=10.21, p<.001\right)$. It was found that group significantly predicted externalising proportion in phase $2(\beta=.28, p<.001)$, whereas accuracy in phase 1 was not a significant predictor.

Last, we were interested in studying whether the use of reminders improves accuracy in phase 2 . We performed a multiple regression with phase-2 accuracy as dependent variable and phase- 1 accuracy and externalising proportion in phase 2 as independent variables. We conducted this analysis separately for the two groups. For the No Reminders group, the two predictors explained a significant amount of the variance in phase-2 accuracy $\left(R^{2}=.30, F(2,93)=20.04, p<.001\right)$. Phase- 1 accuracy significantly predicted accuracy in phase $2(\beta=.44, p<.001)$, as did externalising proportion $(\beta=10.83, p<.001)$. Also for the Forced Reminders group the two predictors explained a significant amount of the variance $\left(R^{2}=.26, F(2,93)=16.29, p<\right.$ $.001)$. Again, phase-1 accuracy significantly predicted accuracy in phase $2(\beta=.40, p<$ $.001)$, as did externalising proportion $(\beta=12.85, p<.001)$.

All analyses above were included in our original pre-registered analysis plan. In an additional post-hoc analysis which was not pre-registered, we explored how reminder setting varied across trials in the two groups (see Figure 5). [Figure 5 approximately here] From visual inspection, it appears that in both groups the use of reminders remained relatively constant over time, being significantly higher for the Forced Reminder group in every trial $(\mathrm{p}<.05)$. This result was confirmed by a further analysis. We fitted individual regression lines predicting the externalising proportion 
from trial number and compared the average slopes in the two groups. The difference between the two slopes was not statistically significant (see Figure $6 ; t(188)=0.14, p=$ $.89, d=0.02)$. [Figure 6 approximately here]

\section{Discussion}

As in the Experiment 1, the use of reminders in phase 1 led to a higher accuracy, confirming the benefit of cognitive aids to reduce memory load and fulfil future intentions.

Another relevant result is the significant correlations between phase-1 accuracy and externalising proportions. For participants in the No Reminders group, objective performance in phase 1 was negatively related to intention offloading in phase 2 , suggesting that the experience of making errors when solving the task using their own unaided memory - reflected in a lower phase-1 performance - may have triggered an increase in phase-2 intention offloading. Conversely, for the Forced Reminder group, objective performance in phase 1 was positively related to intention offloading in phase 2, suggesting that participants who experienced successful performance using an intention offloading strategy were more likely to continue with the same strategy in phase 2 .

As foreseen from the power calculation, we were not able to detect an effect of setting reminders on accuracy in phase 2 . Nevertheless, despite nonsignificant, accuracy in phase 2 was slightly higher for the Forced Reminder group than the No Reminder group. It is possible that an alternative task, manipulating task difficulty, would show a stronger relationship between reminder-use and accuracy.

Furthermore, in line with the main hypothesis, we found that participants who used reminders in the first half of the experiment were significantly more likely to rely on this strategy again when given a free choice, suggesting that once people are in the 
habit of using reminders, they keep using them. Experiment 3 seeks to further look into the mechanisms that might explain this result.

\section{Experiment 3}

In the previous experiment, we found that the use of a cognitive offloading strategy increased the likelihood of relying on such strategy in the future. However, two different factors might explain the results: the experience gained learning to solve the task with a particular strategy or a mere repetition of the last strategy adopted. The main aim of this study was to distinguish between these two possibilities. We did this by repeating the procedure of Experiment 2 with one modification: after phase 1 participants performed a single trial with the opposite strategy before commencing phase 2 where they had free choice over which strategy to use. This means that just before the free-choice trials, participants who learned the task without reminders had used reminders on the previous trial, and vice-versa.

Different results were expected depending on whether participants were biased towards the strategy they used when first learning the task, versus the strategy used on the immediately-preceding trial. We evaluated evidence for two main possibilities:

- If participants were biased to repeat the strategy they used when they first learned the task, the group that used reminders in phase 1 would be more likely to use reminders when given a free choice in phase 2 , despite the reversal-trial between the two phases where the strategies are reversed.

- If participants were simply biased to repeat the strategy used on the previous trial, the opposite effect would be expected, and participants would be more likely to repeat the strategy used on the reversal-trial. 
We also considered the possibility of a combination of these effects, i.e. participants could be influenced both by the initial strategy and the reversal-trial strategy. These effects would be expected to yield opposite influences and could potentially cancel each other out. To evaluate evidence for this possibility we compared results from this experiment to our earlier study. We acknowledge that betweenexperiment comparisons are not as well-controlled as within-experiment comparisons, nevertheless we expected that the between-experiment analyses could provide additional useful information.

The use of reminders may affect not only the likelihood of using them again in the future, but also performance accuracy. We expected to replicate the finding that the use of reminders increases performance accuracy. Accordingly, we hypothesised that in phase 1 participants who are initially instructed to set reminders will be more accurate than participants who are initially asked to solve the task using their own memory. Additionally, we hypothesised that a sufficiently-powered study would find that any factor that increases use of reminders in phase 2 would yield a commensurate increase in accuracy. However, we did not believe that our study was well powered for this particular test, therefore we made no specific predictions about phase-2 accuracy. Before commencing data collection, we preregistered our hypotheses, sample size, experimental procedure, participant exclusion criteria, and analysis plans (https://osf.io/vg6tz/).

\section{Method}

\section{Participants}

A total of 192 participants took part in the experiment (mean age: 35 years, range: 1970; 73 female, 119 male). 
A statistical power analysis was performed with $\mathrm{G}^{*}$ Power 3.1 for sample size estimation. The computations were based on the results of Experiment 2. This revealed an effect size of $d=0.65$ when comparing the proportion of reminder setting between participants who in a first phase always used reminders with those who never did. With an alpha $=.05$ and power $=0.80$, the projected sample size needed with this effect size is approximately $N=76$ (38 participants in each group). However, we expected to find a smaller effect than in the previous study seeing as the reversal-trial manipulation may weaken or even reverse any effect, which would require a larger sample. Mindful of the intention to compare the results with those of the previous study, we decided to keep the same sample size: $N=192$ (96 participants in each group).

We also considered the sample size needed to study accuracy in phase 2 . With the sample size of 192 participants, the comparison of phase- 2 accuracy between the two groups conducted in the previous study had an effect size of $d=0.01$. Since we are planning on using the same sample size, we do not believe that the current study is wellpowered for this comparison, and we are aware that an alternative task where there is a stronger relationship between reminder-use and accuracy might be more appropriate to investigate this question.

Participants were randomly allocated to two groups: Forced Reminders (96 participants) and No Reminders (96 participants). The same exclusion criteria as in the previous experiments were used. Participation took on average 28 minutes $($ minimum $=$ 11 minutes, maximum $=2$ hours 15 minutes), for which participants were paid $\$ 2.50$. All participants provided informed consent before participating and the research was approved by the UCL Research Ethics Committee.

\section{Results}

Mean arithmetic-verification accuracy was 99\%. 


\section{Objective Accuracy}

We first compared accuracy between the two groups in phase 1, using a Welsh twosample t-test. Accuracy was significantly higher in the Forced Reminders group than the No Reminders group, $t(161.7)=3.89, p<.001, d=0.56$ showing, as in the previous studies, that the use of external reminders improves task performance compared with the use of internal memory only (see Figure 3).

Next, we conducted an independent samples t-test comparing accuracy in phase 2 between the two groups. We did not find any significant difference between the two groups, $t(190)=0.21, p=.83, d=0.03$.

Finally, we compared accuracy in each group between phase 1 and phase 2, using a paired t-test. There was a nonsignificant drop in accuracy from phase 1 to phase 2 in the Forced Reminders group $(t(95)=1.02, p=.31, d=0.10)$ and highly significant increase in the No Reminders group $(t(95)=4.71, p<.001, d=0.48)$.

\section{Reminder Setting}

As our main hypothesis-testing analysis, we first compared the likelihood of using reminders in phase 2 in the two conditions. An independent samples t-test found no significant differences in the externalising proportion in the two groups, $t(190)=0.04, p$ $=.97, d=0.01$ (see Figure 4). A Bayesian independent samples t-test was also conducted to investigate the strength of the evidence in favour of our hypothesis against the null hypothesis. An estimated Bayes factor (null/alternative) suggested that the data were 6.37 times more likely to occur under a model without including an effect for experimental condition, rather than the model with it.

As mentioned above, we also considered the possibility that the strategy used in phase 1, as well as the strategy used on the reversal-trial, might both have influenced strategy choice in phase 2 . These effects would operate in opposite directions and could 
potentially cancel each other out leading to a reduced effect of phase-1 strategy on phase- 2 choices, or no effect at all. To explore evidence for this possibility we compared results from this experiment to those of Experiment 2 performing a $2 \times 2$ ANOVA with Condition and Experiment as factors. There was a significant effect of Condition, $F(1,380)=9.57, p=.002, \eta_{\mathrm{p}}{ }^{2}=.025$, reflecting that on average, the externalising proportion was higher in the Forced Reminders condition than in the No Reminders group. There was also a significant interaction between Condition and Experiment, $F(1,380)=9.18, p=.003, \eta_{\mathrm{p}}{ }^{2}=.024$, showing that the influence of initial phase-1 strategy on phase- 2 reminder setting was significantly different between the experiments. The main effect of Experiment was not significant $F(1,380)=0.03, p=$ $.85, \eta_{\mathrm{p}}^{2}=.00$.

Last, we performed an exploratory analysis to compare the externalising proportion in phase 2 across experiments separately for the two groups. For the No Reminders group, we found that the externalising proportion was marginally higher in Experiment $3(M=0.60)$ than in Experiment $2(M=0.47), t(190)=1.93, p=.056, d=$ 0.28. By contrast, we found that for the Forced Reminders group, the externalising proportion was significantly higher in Experiment $2(M=0.74)$ than in Experiment 3 $(M=0.60), t(184.85)=2.39, p=.018, d=0.34$. We also conducted Bayesian independent samples t-tests, which yielded a BF of 1.14 in favour of the null hypothesis for the No Reminders group and a BF of 2.19 for the hypothesis that there is a significant difference between Experiments 2 and 3 for the Forced Reminders group.

\section{Relationship between Accuracy and Reminder Setting}

Next, we ran a correlation between accuracy in phase 1 and reminder choice in phase 2 to investigate whether there is a relationship between performance in phase 1 and choice to set reminders in phase 2. Correlation coefficients were positive in both groups but 
nonsignificant (No Reminders: $r_{\mathrm{s}}(94)=.08, p=.44$; Forced Reminders: $r_{\mathrm{s}}(94)=.12, p$ $=.24)$. We also combined over the two groups and ran a multiple regression where group was a factor. The results of the regression indicated the two predictors explained only $1 \%$ of the variance $\left(R^{2}=.01, F(2,189)=1.43, p=.24\right)$.

Last, we were interested in studying whether the use of reminders improves accuracy in phase 2 . We performed a multiple regression with phase-2 accuracy as dependent variable and phase- 1 accuracy and externalising proportion in phase 2 as independent variables. We conducted this analysis separately for the two groups. For the No Reminders group, the two predictors explained a significant amount of the variance in phase-2 accuracy $\left(R^{2}=.46, F(2,93)=39.64, p<.001\right)$. Phase-1 accuracy significantly predicted accuracy in phase $2(\beta=.46, p<.001)$, as did externalising proportion $(\beta=10.25, p<.001)$. Also for the Forced Reminders group the two predictors explained a significant amount of the variance $\left(R^{2}=.41, F(2,93)=32.42, p<\right.$ $.001)$. Again, phase-1 accuracy significantly predicted accuracy in phase $2(\beta=.70, p<$ $.001)$, whereas externalising proportion was not a significant predictor $(\beta=1.57, p=$ $.54)$.

Along with the pre-registered analyses above, we also conducted an exploratory analysis to examine how reminder setting varied across trials in the two groups (see Figure 5). The graph suggests that participants were initially more likely to rely on the strategy used in the reversal-trial and then returned to the strategy learnt originally in the first phase of the experiment. This result was confirmed by a further analysis. We fitted individual regression lines predicting the externalising proportion from trial number and compared the average slopes in the two groups. The difference between the two slopes was statistically significant (see Figure $6 ; t(188)=2.51, p=.013, d=0.36$. Therefore, even though the groups did not differ significantly in the overall amount of 
reminder setting in phase 2 , they did differ in the temporal profile of strategy choices over the 10 trials.

\section{Discussion}

Whereas phase-1 strategy had a strong effect on phase-2 reminder setting in Experiment 2 , this effect seemed to be eliminated in the present experiment. This suggests that the strategy perseveration effect seen in the earlier experiment can be washed out by performing just one trial of the opposite strategy, or that the influences of phase-1 strategy (in one direction) and reversal-trial strategy (in the other) cancelled each other out. Either way, results indicate that the influence of the strategy used when first learning the task (seen in Experiment 2) can be modulated by the strategy used on a subsequent trial. Furthermore, the single-trial analysis showed that the groups differed in the dynamics of their strategy choices over time. This contrasts with the relatively stable strategy choices seen in Experiment 2. One interpretation of this would be that participants' strategy choices are influenced both by the immediately-preceding trial, and their earlier use of strategies when first learning the task. To test such an account, it would be necessary to manipulate the factors of phase-1 strategy and reversal-trial strategy independently, rather than manipulating them together as in the present experiment.

\section{General Discussion}

The present study investigated the effect of cognitive offloading on A) unaided memory ability, and B) subsequent strategy choice in memory task requiring participants to maintain intentions over a brief delay. External memory is becoming crucial in today's life, but it is also changing how people use their own memory systems (Nestojko, Finley, \& Roediger, 2013). Two alternative arguments can be found in the literature. On 
the one hand, offloading memory onto the environment can overcome the limited capacity of working memory, relieve the burden on prospective memory, and improve cognitive functioning (Finley et al., 2018). On the other hand, externally stored information may not always be a viable replacement for internal memory and excessive reliance on external memory sources may reduce flexible access to internally stored knowledge, impairing in turn higher order cognition (Nestojko et al., 2013). Similar concerns date back at least to the time of Socrates, who worried over 2000 years ago that the use of writing would "introduce forgetfulness into the soul of those who learn it: they will not practice using their memory because they will put their trust in writing" (Plato, approximately $370 \mathrm{BC} / 1995$, p. 79). To date, despite widespread interest in these issues (e.g., "Is Google making us stupid?"; Carr, 2008), relatively little empirical data has been collected to address the two possibilities outlined above. We therefore conducted the present study to collect further evidence in the context of intention offloading to better understand the effect that the use of external reminders has on unaided memory ability and subsequent strategy choice.

The first aim of this study was to investigate the influence of earlier offloading on subsequent unaided memory ability. Some previous research has suggested that the act of cognitive offloading may have a detrimental effect on unaided memory for the specific memories that are offloaded (Henkel, 2014; Soares \& Storm, 2018; Tamir et al., 2018). Other studies suggest that offloading memory to an external store can improve individuals' ability to encode and remember subsequently-presented information (Storm $\&$ Stone, 2015). Here, we found no significant impact of earlier offloading on subsequent performance. We suggest that the key difference between our study and the earlier ones is as follows. In studies that report a detrimental effect of offloading on memory (Henkel, 2014; Soares \& Storm, 2018; Tamir et al., 2018), participants were 
tested on their unaided recall of the very information that was either offloaded or maintained internally. Impaired memory for the offloading information might then be explained by participants dismissing information from internal memory which they believe to be stored externally (Henkel, 2014), or interference to memory encoding caused by the act of offloading (Soares \& Storm, 2018).

In studies that report an improvement to memory caused by offloading (Storm \& Stone, 2015), participants are tested on their unaided memory for new information acquired whilst still maintaining earlier information that was either offloaded or not. In both cases, the information that was originally offloaded or maintained internally continued to be task-relevant at the time of the main hypothesis-testing memory test. By contrast, our experiment compared two conditions where the earlier information was no longer required when the hypothesis-testing memory test took place, regardless of whether it was originally offloaded or maintained internally. Our results suggest that in these circumstances, short-term use of an offloading or internal strategy has little effect on subsequent unaided memory performance. Of course, it is quite possible that longerterm or habitual use of an offloading or internal memory strategy over an extended period of time might impact on unaided memory abilities. Investigating this question would require a different research design to the one used here.

Furthermore, the three experiments here reported replicated the finding that cognitive offloading can increase performance accuracy (e.g., Gilbert, 2015b; Storm \& Stone, 2015). This points to an important factor in the interplay between internal and external memory. While much of the ongoing debate since the time of Socrates has focused on whether or not offloading has a detrimental effect on our unaided ability, it should also be recognised that not engaging in cognitive offloading could also have a 
detrimental effect on our performance, in that it can impair subsequent strategy choice, in contexts where an offloading strategy is available and effective.

The second aim of the current study was to address this issue, by testing whether individuals' strategy choice is affected by previous strategies used in the context of an intention offloading task. The results of Experiment 2 showed that once participants were in the habit of using reminders, they continued to rely on them when given a free choice. The findings align with previous research documenting the so-called Einstellung effect (Luchins, 1942) or perseveration effect (Schillemans et al., 2010) showing that the repeated implementation of a strategy increases the likelihood of using the same strategy again. This effect might explain why in the second phase of the experiment participants in both conditions were biased towards the strategy used in the earlier phase. That is, this mechanism made participants blind to the possibility of using another strategy, even a more efficient one, such as switching to the use of reminders for the No Reminders group. Support for this interpretation comes from the literature on the priming effect - that is, the recent use of a specific cognitive strategy leads to a temporary increase in the likelihood of applying the same procedure in a subsequent task (Higgins, 1996). As a consequence, when given a free choice in phase 2 , participants might have favoured the primed strategies - i.e., the one used in phase 2 - at the expense of the other one.

Furthermore, there is evidence showing that after switching strategy in a cognitive task, participants exhibit longer reaction times and possibly higher error rates than when repeating the same strategy (Lemaire \& Lecacheur, 2010; Luwel, Schillemans, Onghena, \& Verschaffel, 2009). It is possible that in the second phase of experiment 2 participants continued to apply the strategy learnt in phase 1 in the attempt to avoid such a strategy-switch cost. As noted by Schillemans and colleagues (2012), in 
certain situations, such as when different strategies are almost equally valid, continuing to apply the same strategy might be more adaptive than switching to another one because the strategy-switch cost may be higher than the benefit obtained by the use of the more efficient strategy. This might explain why participants in the No Reminders group kept solving the task using their own memory, even though switching to the use of reminders could have been more effective. Further research might be conducted manipulating the difficulty level of the task to study the threshold between strategyswitch cost and reminder-setting benefits to further understand these mechanisms. The manipulation of task difficulty seems to be a good avenue also to further investigate the influence of strategy perseveration on task performance accuracy, for which this study was not well powered.

It is unclear from the results of Experiment 2 whether participants were biased to repeat the strategy they used when first learning the task, or simply the strategy performed on the previous trial. We therefore conducted a third experiment in which we added a strategy reversal trial between the two phases. This aligns to the existing research investigating the number of trials needed to observe the perseveration effect and potential differences in the strength of the effect according to the number of preceding trials (Schillemans et al., 2012). This research suggests that a single application of a certain strategy can be sufficient to create a perseveration effect and bias participants' strategy choices on subsequent trials (Lemaire \& Lecacheur, 2010; Schillemans et al., 2012). Consistent with this, experiment 3 showed that a single reversal-trial between phases 1 and 2 eliminated the group difference in the overall number of reminders set in phase 2 of the task. However, the single-trial analysis showed that the groups still differed in the temporal profile of strategy choices over the course of 10 trials. These findings suggest that a brief intervention, potentially involving 
just a single application of a particular strategy, could have a substantial effect on individuals' subsequent decisions whether to engage in cognitive offloading. However, the effect of such an intervention may fade over time.

Despite the increasing interest in cognitive offloading over recent years, many questions remain regarding the factors that affect the likelihood of choosing to offload cognition rather than rely on internal memory. A promising avenue for future research is the investigation of individual differences on offloading strategies. As pointed out by Boldt and Gilbert (Boldt \& Gilbert, 2019), cognitive offloading is idiosyncratic in that different individuals use different aids to support their cognitive acts and different cognitive aids might be more appropriate than others in certain situations. The paradigm adopted in this study investigates a very specific cognitive offloading strategy, which might not be optimal for every individual and the online nature of the study limits the control over the strategy used by participants. Individual characteristics such as rigidity might strengthen the bias towards the repeated application of a previously implemented strategy (Schillemans et al., 2012). This is particularly relevant when considering how age interacts with the effect of strategy history on cognitive offloading. Research has shown that individuals become more rigid in later adulthood (Lemaire, Arnaud, \& Lecacheur, 2004; Lemaire \& Lecacheur, 2001), and this rigidity may fuel the bias towards the repeated application of the strategy used on previous items. Further delineating how age impacts upon the perseveration effect and on the decision to use a cognitive offloading strategy rather than relying on the internal memory represents an important direction for further research.

On a theoretical level, the present results are relevant for the understanding of the interrelation between internal and external memory, and the delineation of an additional factor affecting cognitive offloading, namely the influence of the previously 
used strategy. Past strategy history has to be taken into consideration in theoretical accounts of the mechanism underlying people's strategy choice and the decision to rely on cognitive offloading strategies rather than using their own memory.

\section{Conclusion}

Technological advancement has profoundly changed how we access and store information. The possibility to offload into the environment more than we used to in the past offers the opportunity to reduce the burden of intention maintenance in internal memory. However, the consequences of this for future cognitive performance are not well understood. Our results suggest that short-term use of a cognitive offloading strategy has little influence on subsequent unaided performance, in a context where the information that was originally offloaded or stored internally is no longer relevant. However, there can be strong effects of prior offloading on subsequent strategy choice. In sum, we find little evidence for a risk that short-term cognitive offloading can impair subsequent unaided ability. However, there may be a risk that not engaging in cognitive offloading can impair subsequent strategy choice, in contexts where an offloading strategy is available and effective.

Acknowledgements: This work was supported by the Economic and Social Research Council (ESRC) under Grant ES/N018621/1.

Disclosure statement: The authors declare that they do not have competing interests.

\section{References}

Baldwin, V. N., Powell, T., \& Lorenc, L. (2011). Factors influencing the uptake of memory compensations: A qualitative analysis. Neuropsychological Rehabilitation, 21(4), 484-501. https://doi.org/10.1080/09602011.2011.582378 
Baumeister, R. F., Bratslavsky, E., Muraven, M., \& Tice, D. M. (1998). Ego depletion: Is the active self a limited resource? Journal of Personality and Social Psychology, 74(5), 1252-1265. https://doi.org/10.1037/0022-3514.74.5.1252

Bilalić, M., McLeod, P., \& Gobet, F. (2008). Inflexibility of experts—Reality or myth? Quantifying the Einstellung effect in chess masters. Cognitive Psychology, 56(2), 73-102. https://doi.org/10.1016/J.COGPSYCH.2007.02.001

Boldt, A., \& Gilbert, S. (2019). Confidence Guides Spontaneous Cognitive Offloading. https://doi.org/10.31234/OSF.IO/CT52K

Carr, N. G. (2008). Is Google making us stupid? Boston, MA: The Atlantic.

Cowan, N. (2010). The magical mystery four: How is working memory capacity limited, and why? Current Directions in Psychological Science, 19(1), 51-57. https://doi.org/10.1177/0963721409359277

Crump, M. J. C., McDonnell, J. V., \& Gureckis, T. M. (2013). Evaluating Amazon's Mechanical Turk as a tool for experimental behavioral research. PLoS ONE, 8(3), e57410. https://doi.org/10.1371/journal.pone.0057410

Dang, J. (2018). An updated meta-analysis of the ego depletion effect. Psychological Research, 82(4), 645-651. https://doi.org/10.1007/s00426-017-0862-x

Einstein, G. O., \& McDaniel, M. A. (1990). Normal aging and prospective memory. Journal of Experimental Psychology. Learning, Memory, and Cognition, 16(4), 717-726. https://doi.org/http://dx.doi.org/10.1037/0278-7393.16.4.717

Ellis, J. J., \& Reingold, E. M. (2014). The Einstellung effect in anagram problem solving: evidence from eye movements. Frontiers in Psychology, 5, 679. 
https://doi.org/10.3389/fpsyg.2014.00679

Ferguson, A. M., McLean, D., \& Risko, E. F. (2015). Answers at your fingertips: Access to the Internet influences willingness to answer questions. Consciousness and Cognition, 37, 91-102. https://doi.org/10.1016/j.concog.2015.08.008

Finley, J. R., Naaz, F., \& Goh, F. W. (2018). Memory and Technology: How We Use Information in the Brain and the World. Springer, Cham.

Fisher, M., Goddu, M. K., \& Keil, F. C. (2015). Searching for explanations: How the Internet inflates estimates of internal knowledge. Journal of Experimental Psychology: General, 144(3), 674-687. https://doi.org/10.1037/xge0000070

Friese, M., Loschelder, D. D., Gieseler, K., Frankenbach, J., \& Inzlicht, M. (2018). Is ego depletion real? An analysis of arguments. Personality and Social Psychology Review, 1-25. https://doi.org/10.1177/1088868318762183

Gilbert, S. J. (2015a). Strategic offloading of delayed intentions into the external environment. Quarterly Journal of Experimental Psychology, 68(5), 971-992. https://doi.org/10.1080/17470218.2014.972963

Gilbert, S. J. (2015b). Strategic use of reminders: Influence of both domain-general and task-specific metacognitive confidence, independent of objective memory ability. Consciousness and Cognition, 33, 245-260. https://doi.org/10.1016/j.concog.2015.01.006

Gilbert, S. J., Bird, A., Carpenter, J., Fleming, S., Sachdeva, C., \& Tsai, P.-C. (in press). Optimal use of reminders: Metacognition, effort, and cognitive offloading. Ournal of Experimental Psychology: General. https://doi.org/DOI: 10.1037/xge0000652 
Graf, P., \& Uttl, B. (2001). Prospective Memory: A New Focus for Research.

Consciousness and Cognition, 10(4), 437-450.

https://doi.org/10.1006/ccog.2001.0504

Hagger, M. S., Chatzisarantis, N. L. D., Alberts, H., Anggono, C. O., Batailler, C., Birt, A. R., ... Zwienenberg, M. (2016). A multilab preregistered replication of the egodepletion effect. Perspectives on Psychological Science, 11(4), 546-573.

https://doi.org/10.1177/1745691616652873

Henkel, L. A. (2014). Point-and-shoot memories: The influence of taking photos on memory for a museum tour. Psychological Science, 25(2), 396-402. https://doi.org/10.1177/0956797613504438

Herrmann, D., Brubaker, B., Yoder, C., Sheets, V., \& Tio, A. (1999). Devices that remind. In F. Durso, R. Nickerson, R. Schvaneveldt, S. Dumais, D. Lindsay, \& M. Chi (Eds.), Handbook of applied cognition (1st ed., pp. 377-407). New York: Wiley.

Higgins, E. T. (1996). Knowledge activation: Accessibility, applicability, and salience. In E. T. Higgins \& A. W. Kruglanski (Eds.), Social Psychology: Handbook of Basic Principles (pp. 133-168). NewYork, NY: The Guilford Press.

Jeffreys, H. (1961). Theory of probability (3rd ed.). Oxford, UK: Oxford University Press.

Lemaire, P., Arnaud, L., \& Lecacheur, M. (2004). Adults' age-related differences in adaptivity of strategy choices: Evidence from computational estimation. Psychology and Aging, 19(3), 467-481. https://doi.org/10.1037/08827974.19.3.467 
Lemaire, P., \& Lecacheur, M. (2001). Older and younger adults' strategy use and execution in currency conversion tasks: insights from French franc to euro and euro to French franc conversions. Journal of Experimental Psychology. Applied, 7(3), 195-206. Retrieved from http://www.ncbi.nlm.nih.gov/pubmed/11676098

Lemaire, P., \& Lecacheur, M. (2010). Strategy switch costs in arithmetic problem solving. Memory \& Cognition, 38(3), 322-332.

https://doi.org/10.3758/MC.38.3.322

Luchins, A. S. (1942). Mechanization in problem solving: The effect of Einstellung. Psychological Monographs, 54(6), i-95. https://doi.org/10.1037/h0093502

Lurquin, J. H., \& Miyake, A. (2017). Challenges to ego-depletion research go beyond the replication crisis: A need for tackling the conceptual crisis. Frontiers in Psychology, 8, 568. https://doi.org/10.3389/fpsyg.2017.00568

Luwel, K., Schillemans, V., Onghena, P., \& Verschaffel, L. (2009). Does switching between strategies within the same task involve a cost? British Journal of Psychology, 100(4), 753-771. https://doi.org/10.1348/000712609X402801

Macias, C., Yung, A., Hemmer, P., \& Kidd, C. (2015). Memory strategically encodes externally unavailable information. In D. C. Noelle, R. Dale, A. S. Warlaumont, J. Yoshimi, T. Matlock, C. D. Jennings, \& P. P. Maglio (Eds.), Proceedings of the 37th Annual Meeting of the Cognitive Science Society. Austin, TX. Retrieved from https://mindmodeling.org/cogsci2015/papers/0255/paper0255.pdf

Nestojko, J. F., Finley, J. R., \& Roediger, H. L. (2013). Extending cognition to external agents. Psychological Inquiry, 24, 321-325.

https://doi.org/10.1080/1047840X.2013.844056 
Plato. (1995). Phaedrus. (A. Nehamas \& P. Woodruff, Trans.). Indianapolis, IN: Hackett Publishing Company. (Original work composed around 370 BC).

Risko, E. F., \& Gilbert, S. J. (2016). Cognitive Offloading. Trends in Cognitive Sciences, 20(9), 676-688. https://doi.org/10.1016/J.TICS.2016.07.002

Runge, Y., Frings, C., \& Tempel, T. (2019). Saving-enhanced performance: Saving items after study boosts performance in subsequent cognitively demanding tasks. Manuscript Submitted for Publication.

Schillemans, V., Luwel, K., Bulté, I., Onghena, P., \& Verschaffel, L. (2010). The influence of previous strategy use on individuals' subsequent strategy choice: Findings from a numerosity judgement task. Psychologica Belgica, 49(4), 191. https://doi.org/10.5334/pb-49-4-191

Schillemans, V., Luwel, K., Ceulemans, E., Onghena, P., \& Verschaffel, L. (2012). The effect of single versus repeated previous strategy use on individuals' subsequent strategy choice. Psychologica Belgica, 52(4), 307. https://doi.org/10.5334/pb-52-4307

Smith, R. E. (2003). The cost of remembering to remember in event-based prospective memory: Investigating the capacity demands of delayed intention performance. Journal of Experimental Psychology: Learning, Memory, and Cognition, 29(3), 347-361. https://doi.org/10.1037/0278-7393.29.3.347

Soares, J. S., \& Storm, B. C. (2018). Forget in a flash: A further investigation of the photo-taking-impairment effect. Journal of Applied Research in Memory and Cognition, 7(1), 154-160. https://doi.org/10.1016/J.JARMAC.2017.10.004 
Storm, B. C., \& Stone, S. M. (2015). Saving-enhanced memory: The benefits of saving on the learning and remembering of new information. Psychological Science, 26(2), 182-188. https://doi.org/10.1177/0956797614559285

Storm, B. C., Stone, S. M., \& Benjamin, A. S. (2016). Using the Internet to access information inflates future use of the Internet to access other information. https://doi.org/10.1080/09658211.2016.1210171

Tamir, D. I., Templeton, E. M., Ward, A. F., \& Zaki, J. (2018). Media usage diminishes memory for experiences. Journal of Experimental Social Psychology, 76, 161-168. https://doi.org/10.1016/J.JESP.2018.01.006

Ward, A. F. (2013). One with the Cloud: Why People Mistake the Internet's Knowledge for Their Own. Doctoral Dissertation, Harvard University. Retrieved from http://nrs.harvard.edu/urn-3:HUL.InstRepos:11004901 


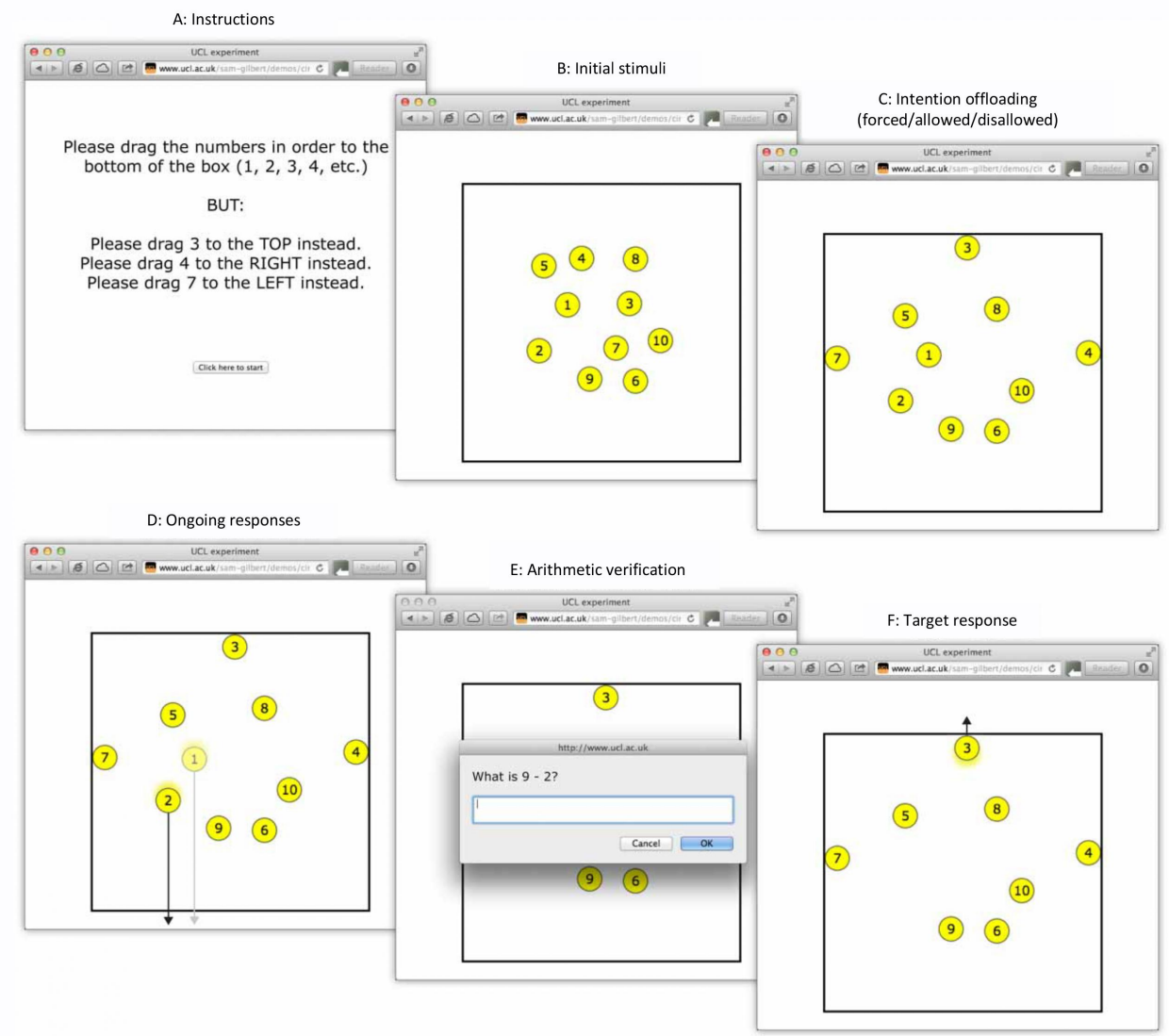

Figure 1. Schematic illustration of the intention-offloading task.

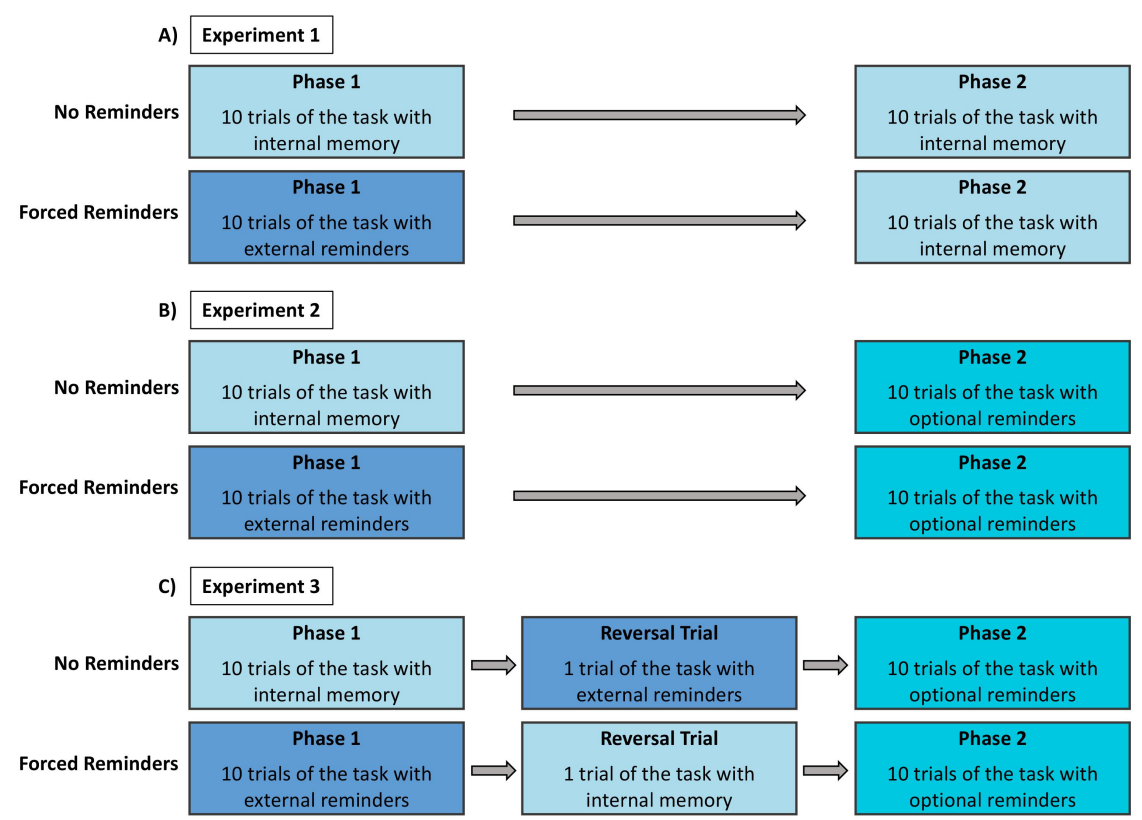

Figure 2. Experimental design for experiment 1 (A), experiment 2 (B) and experiment 3 (C). 

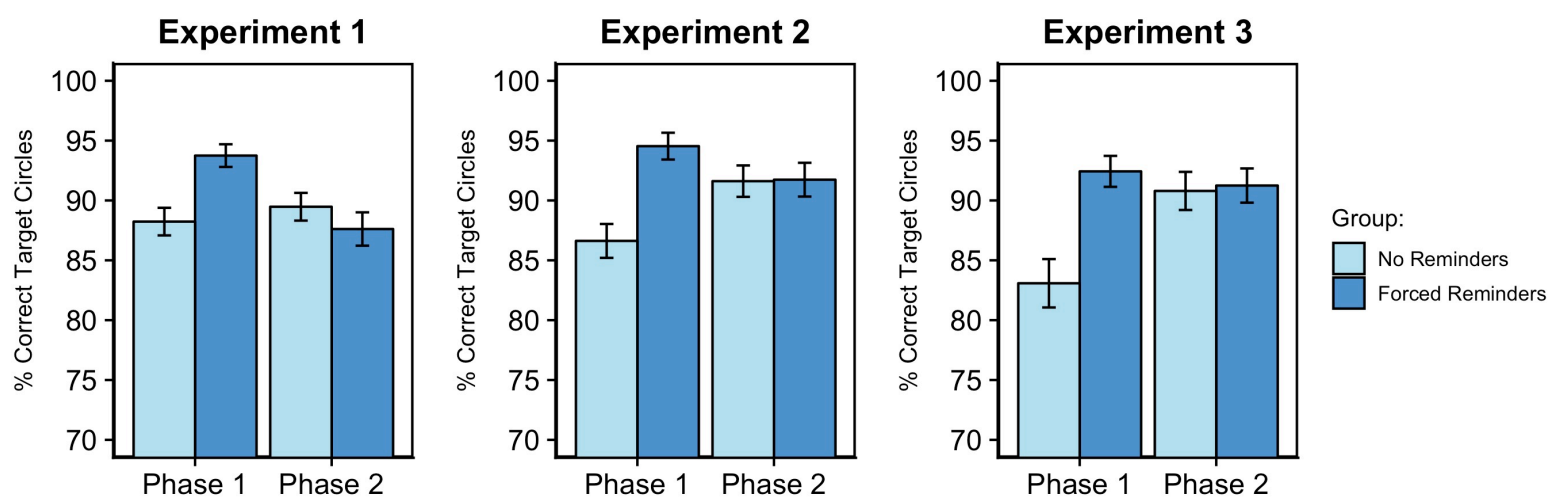

Figure 3. Mean target accuracy in experiments 1, 2, and 3. Error bars indicate standard error of the mean.

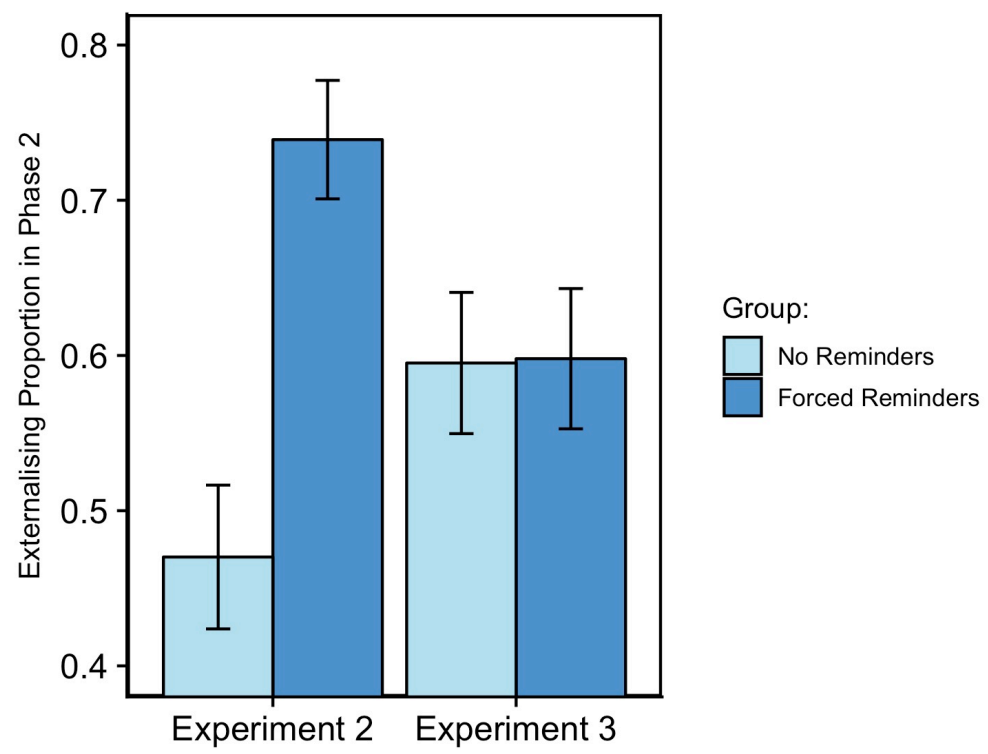

Figure 4. Mean externalising proportion in experiments 2 and 3. Error bars indicate standard error of the mean. 

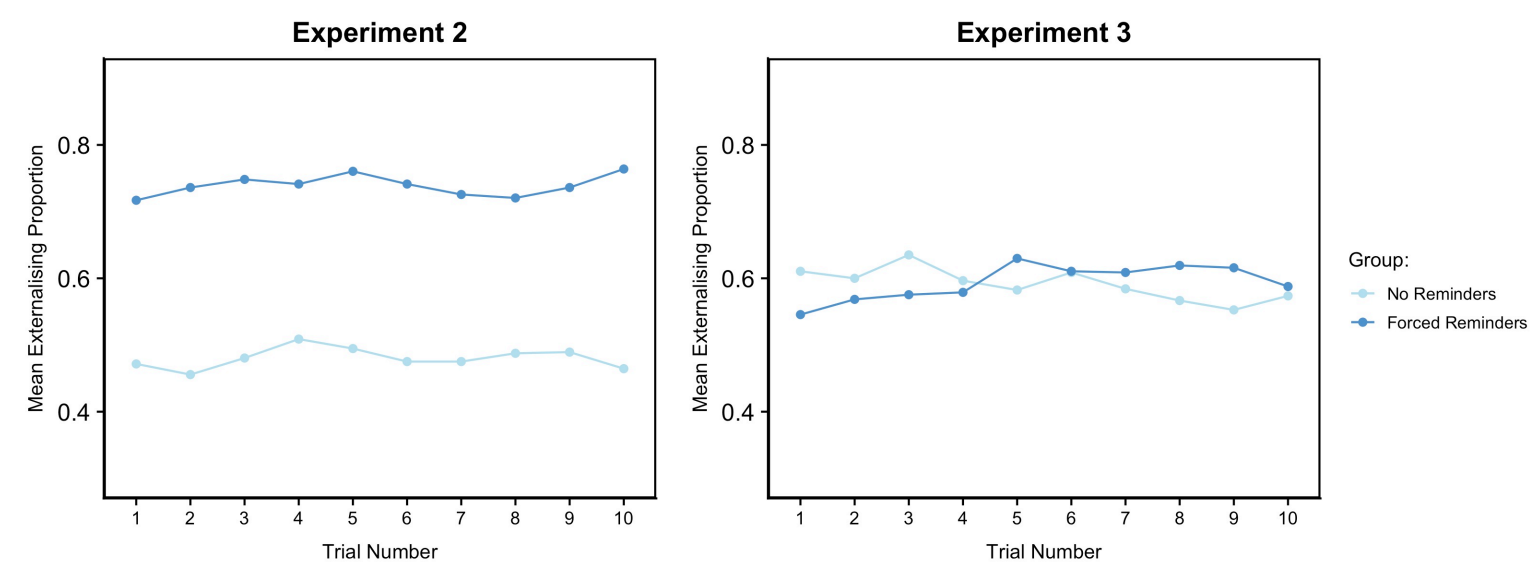

Figure 5. Mean externalising proportion across trials in phase 2 for experiment 2 and experiment 3 .
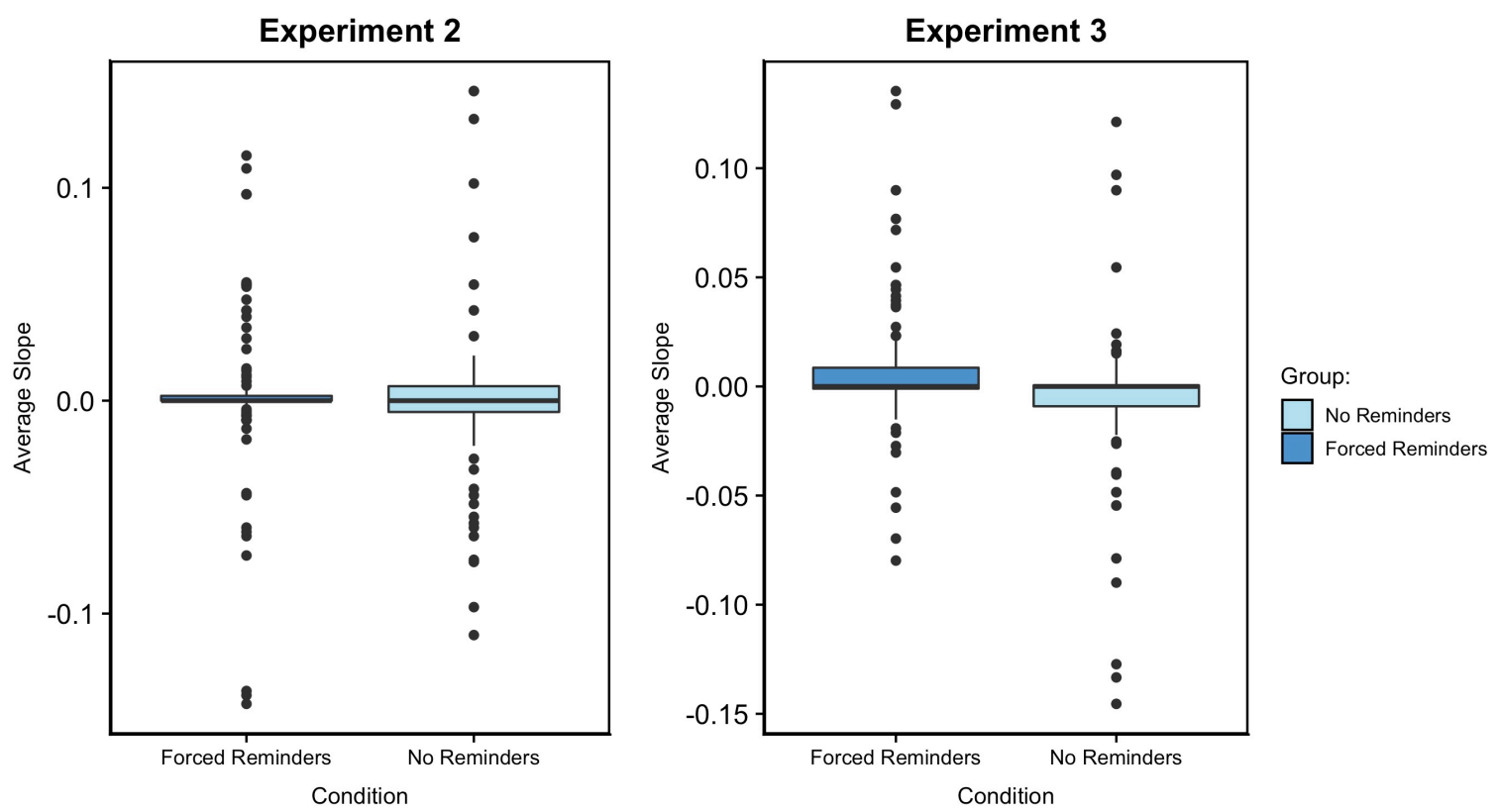

Figure 6. Group-wise distributions of individual slopes from a regression model in which trial predicts the externalising proportion for experiment 2 and experiment 3 . The boxplots reflect the interquartile range (IQR) and the median. The whiskers span from the first quartile minus 1.5 times the IQR to the third quartile plus 1.5 times the IQR. Data points outside this inner fence are shown as individual data points. 